\title{
Editorial: Wrapping Up 2021 and Looking Forward to 2022
}

\author{
Stephen Scypinski ${ }^{1}$
}

Accepted: 16 November 2021 / Published online: 29 November 2021

(c) The Author(s), under exclusive licence to Springer Science+Business Media, LLC, part of Springer Nature 2021

Dear Readers of the Journal of Pharmaceutical Innovation:

As I have reflected on my editorials since taking over as the Editor-in-Chief, it seems that most of them have been centered around the COVID-19 pandemic and its effect on the pharmaceutical industry. Indeed, even as the world moves toward what will be our new normal, COVID seems to be a dominant factor in our professional and personal society. I expect this to continue for the foreseeable future.

It has been an interesting several months since the last issue of JoPI. I was very happy to attend the recent American Association Pharmaceutical Scientists (AAPS) PharmSci 360 meeting in person in Philadelphia, PA, last October. The meeting was conducted as a "hybrid" between in-person and virtual attendees. For those attending in person, there were protocols that had to be followed; however, it felt great to sit in a live session and stroll the exhibition floor and speak to exhibitors. Thank you AAPS. Well done indeed!

Enough on the professional front. It is no secret that the ripple effect of the COVID-19 pandemic has created a supply chain crisis for pharmaceutical companies and their contract organizations. This supply chain pinch has also affected each one of us in our personal lives in areas such as the availability of holiday gifts and yuletide food. A recent article in Contract Pharma highlights the effect of supply chain shortage on pharmaceutical manufacturing. To the best of my knowledge, no critical patient medicines have been delayed due to the pandemic; however, some clinical trials have been delayed and this relates to the next generation of pharmaceutical products. Governments around the globe are taking steps to improve supply chain efficiency, and time will tell if our pharmaceutical conveyor belt returns to normal. I certainly hope so.

One supply chain that has not been interrupted by the pandemic is the steady stream of submitted manuscripts to the Journal of Pharmaceutical Innovation, of which I am very grateful for. Our new issue once again highlights new and improved approaches for formulating active drugs to treat serious afflictions. There are also multivariate and other mathematical designs that seek to improve the drug development process. I am confident that as you peruse this latest issue of the Journal of Pharmaceutical Innovation, you will find materials germane to your work, be it in industry, academia, or private laboratory. Ideas? Please write me at sscypinskli@ comcast.net. Until our next issue in 2022, stay safe and well. Happy Holidays to all!

Stephen Scypinski.

Publisher's Note Springer Nature remains neutral with regard to jurisdictional claims in published maps and institutional affiliations.

Stephen Scypinski

sscypinski@comcast.net

$1 \quad$ VBI Vaccines Inc., Cambridge, MA, USA 